This item was submitted to Loughborough's Research Repository by the author.

Items in Figshare are protected by copyright, with all rights reserved, unless otherwise indicated.

\title{
Continuous disinfection of Fusarium oxysporum in nutrient solution by pulsed electric field
}

PLEASE CITE THE PUBLISHED VERSION

https://doi.org/10.1109/ICHVE49031.2020.9279741

PUBLISHER

IEEE

VERSION

AM (Accepted Manuscript)

\section{PUBLISHER STATEMENT}

(c) 2020 IEEE. Personal use of this material is permitted. Permission from IEEE must be obtained for all other uses, in any current or future media, including reprinting/republishing this material for advertising or promotional purposes, creating new collective works, for resale or redistribution to servers or lists, or reuse of any copyrighted component of this work in other works.

\section{LICENCE}

All Rights Reserved

\section{REPOSITORY RECORD}

Chen, R, Di Zhang, Ninghui Zhu, Chengcheng Liu, Bucur Novac, and Chongshan Zhong. 2020. "Continuous Disinfection of fusarium Oxysporum in Nutrient Solution by Pulsed Electric Field”. Loughborough University. https://hdl.handle.net/2134/13668938.v1. 


\section{Continuous Disinfection of Fusarium oxysporum in Nutrient Solution by Pulsed Electric Field}

\author{
$1^{\text {st }}$ Ran Chen \\ College of Information and Electrical \\ Engineering \\ China Agricultural University \\ Beijing, China \\ ranchen@cau.edu.cn \\ $4^{\text {th }}$ Chengcheng Liu \\ College of Information and Electrical \\ Engineering \\ China Agricultural University \\ Beijing, China \\ 18755881805@163.com
}

\author{
$2^{\text {nd }}$ Di Zhang \\ College of Information and Electrical \\ Engineering \\ China Agricultural University \\ Beijing, China \\ zhangdi_JD@cau.edu.cn \\ $5^{\text {th }}$ Bucur Novac \\ School of Mechanical, Electrical and \\ Manufacturing Engineering \\ Loughborough University \\ Leicestershire, UK \\ b.m.novac@lboro.ac.uk
}

\author{
$3^{\text {rd }}$ Ninghui Zhu \\ C-EPRI Electric Power Engineering \\ Co., Ltd \\ State Grid \\ Beijing, China \\ zhuninghui@163.com \\ $6^{\text {th }}$ Chongshan Zhong* \\ College of Information and Electrical \\ Engineering \\ China Agricultural University \\ Beijing, China \\ zhongchsh@cau.edu.cn
}

\begin{abstract}
The disinfection of recirculating nutrient solution is essential to avoid disasters due to the dispersal of pathogens in a closed soilless culture system. In this work, a disinfection technique using pulsed electric field (PEF) has been investigated. A system was designed to implement continuous disinfection treatment of nutrient solution. The system mainly includes two parts: 1) a pulsed power generator that produces repetitive pulses with amplitude of between $0 \mathrm{kV}$ and $5 \mathrm{kV}$ with a pulse duration of $10 \mu$ s and a pulse repetition frequency of $50 \mathrm{~Hz}$ and 2) a parallel-plate treatment chamber used to realize continuous processing. In order to verify the efficacy of PEF treatment Fusarium oxysporum, being amongst the most destructive root-borne diseases, was selected as the object of study and therefore the nutrient solution was inoculated with this pathogen. The results indicate that PEF treatment deactivated most of the Fusarium oxysporum in the nutrient solution, achieving a maximum disinfection efficiency of $99 \%$. At the same time, no obvious changes of the inorganic compositions in the PEF-treated nutrient solution were observed. The overall system is simple and convenient to be operated, worked stably and was capable of achieving a continuous treatment. This work therefore clearly demonstrates the feasibility of continuous PEF disinfection treatment in real soilless culture systems, opening the door for implementation in various agricultural applications.
\end{abstract}

Keywords—pulsed electric field, nutrient solution, Fusarium oxysporum, sterilization, soilless culture system

*Corresponding Author,Email: zhongchsh@cau.edu.cn

\section{INTRODUCTION}

The recycling of nutrient solutions in soilless culture systems may not only greatly save water and fertilizer but also reduce pollution on rivers and groundwater. However, there is a risk that root-borne diseases could affect closed systems, as pathogens may rapidly spread through recycling nutrient solution and lead to plant infection and even devastating losses ${ }^{[1,2]}$. Therefore, placing an in-situ disinfecting nutrient solution is essential. Several methods have been applied for the disease control in recirculating soilless culture systems such as: ozone, ultraviolet, filtration, chemical sterilization and radiation and biological control ${ }^{[3-8]}$.

Even though ozone disinfection is commonly used, studies indicated that the inactivation ability of ozonized water for Fusarium oxysporum conidia will diminish when the concentration of ozone in water is below $0.1 \mathrm{ppm}^{[9]}$. Besides, for the low solubility in water $\left(0.105 \mathrm{~g} / 100 \mathrm{ml}\right.$ at $\left.0{ }^{\circ} \mathrm{C}\right)$, a significant percentage of ozone may escape from the solution, creating hazard pollution for both people and plants. Applying $\mathrm{UV}$ radiation is another important disinfection technique, but to maintain its efficacy it requires the transparency of nutrient solution to be at least $60 \%{ }^{[10]}$. Moreover, the limited lifetime of UV lamps indirectly increases the cost of equipment. Although the chemical treatment could achieve satisfactory efficacy, the residues also raise concerns for consumers. Therefore, it is desired to develop a safe and effective alternative disinfection method.

The application of pulsed electric field (PEF) has been widely reported in food engineering. Numerous studies indicated that PEF technology is an effective non-thermal sterilization method for liquid food ${ }^{[11]}$. The commonly accepted mechanism of PEF treatment is electroporation on the cell membrane of the pathogen $[12,13]$. Compared to traditional sterilization technologies, PEF has several attractive advantages: higher efficiency in breaking the cell membrane, thorough disinfection, quick treatment, lower temperature rise and therefore less energy consumption ${ }^{[14]}$. Hence, PEF has the potential to act as an alternative nutrient solution disinfection technique for a closed soilless culture system.

Our previous work presented that by the use of PEF treatment, the maximum disinfection efficiency of Fusarium oxysporum in a nutrient solution could reach $99.84 \%$ within a few seconds of application ${ }^{[15]}$. However, the process was static and therefore unable to treat the nutrient solution while in a continuous flow. Additionally, the pulsed generator then used included a spark gas switch which had a short service life and was therefore not practical in real applications.

To demonstrate continuous disinfection of nutrient solution by the PEF technique, a pulsed power generator equipped with a high-voltage semiconductor switch (thyristor) and a novel corresponding treatment chamber were both developed. Several experiments were conducted to show its potential for future practical applications.

\section{MATERIALS AND METHODS}

\section{A. Preparation of plant pathogenic fungal and suspensions}

Fusarium oxysporum was used as the target fungal to evaluate disinfection performance, which was preserved and provided by the college of plant protection, China Agricultural University. Fusarium oxysporum was cultured on potato dextrose agar (PDA) in an incubator at $28^{\circ} \mathrm{C}$ for 5 days. Several mycelial plugs from the edge of the colony were 
transferred to $250 \mathrm{~mL}$ flasks containing potato dextrose broth (PDB) and incubated at $28{ }^{\circ} \mathrm{C}$ on a rotary shaker at a speed of $160 \mathrm{r} / \mathrm{min}$. After 5 days, the liquid was passed through two layers of sterile cheesecloth to filter out tiny fragments of hyphae. The microconidia were counted under a microscope at approximately $1.0 \times 10^{7} \quad$ spores $/ \mathrm{mL}$ using a hemocytometer ${ }^{[16]}$. The fungal solutions were suspended in a hydroponic nutrient solution (approximately $1.0 \times 10^{5} \mathrm{spores} / \mathrm{mL}$ or $\mathrm{CFU} / \mathrm{mL}$ ), which was composed of $370 \mathrm{mg} / \mathrm{L}$ potassium nitrate, $115 \mathrm{mg} / \mathrm{L}$ ammonium dihydrogen phosphate, $483 \mathrm{mg} / \mathrm{L}$ magnesium sulfate, $820 \mathrm{mg} / \mathrm{L}$ calcium nitrate, $25 \mathrm{mg} / \mathrm{L}$ mixed trace elements, respectively. The $\mathrm{pH}$ and electrical conductivity (EC) of the nutrient solution were 6.6 and $1.7 \mathrm{mS} / \mathrm{cm}$, respectively.

\section{B. PEF treatment system}

Fig. 1 demonstrates the experimental arrangement, which consists of a HV pulsed power generator, a treatment chamber, and the necessary monitoring and controlling devices.

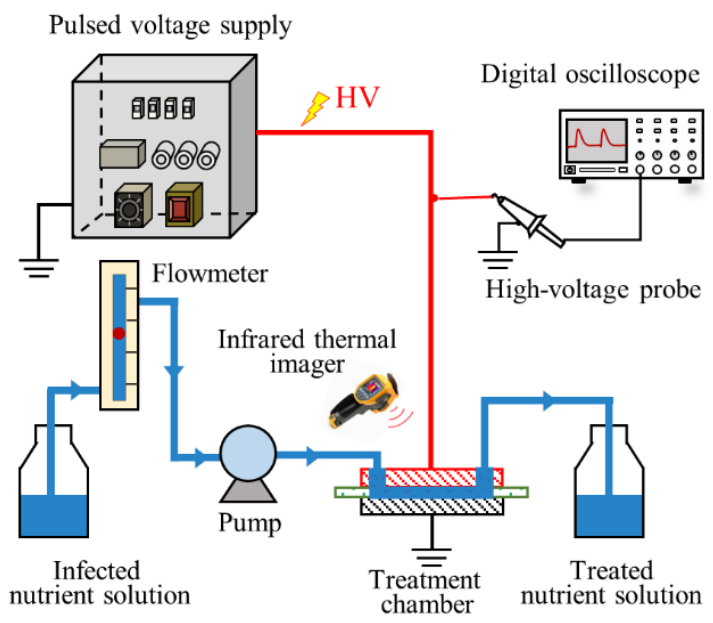

Fig. 1. Experimental arrangement.

Fig. 2 shows the circuit of the HV pulsed power generator, which was designed to generate microsecond output voltage pulses to the load $R_{L}$ i.e., the equivalent resistance of the treatment chamber. The AC power $(220 \mathrm{~V}, 50 \mathrm{~Hz})$ is boosted by a HV transformer. The capacitor $C$ is charged via a diode $D$ in the positive half cycle of voltage and discharged in the negative half cycle. A high-voltage thyristor (5STP08F6500, ABB) is used to switch pulses. A weak underdamped voltage waveform is necessary to maintain the reliable turn-off operation of the thyristor, with the relationship among $R_{L}, L$ and $C$ was given by (1), and the inductance $L$ and capacitance $C$ being $11.5 \mu \mathrm{H}$ and $305 \mathrm{nF}$, respectively. Consequently, the $R_{L}$ is $9.8 \Omega$.

$$
R_{L}=1.6 \sqrt{L / C}
$$

Fig. 3 displays a typical waveform of the pulsed voltage applied to the treatment chamber, measured by a high-quality HV probe (1000:1, P6015A, Tektronix, USA) connected to a digital oscilloscope (TDS2012C, Tektronix, USA). The pulse has a duration approximately equal to $10 \mu$ s and a repetition frequency of $50 \mathrm{~Hz}$. For parallel plate electrodes placed a small distance $d$ apart, the strength of the pulsed electric field can be simply calculated using (2), with the output voltage amplitude $U$ of the generator adjusted between 0 to $5 \mathrm{kV}$, corresponding to an electric field strength $E$ ranging from 0 to $18.5 \mathrm{kV} / \mathrm{cm}$.

$$
E=U / d
$$

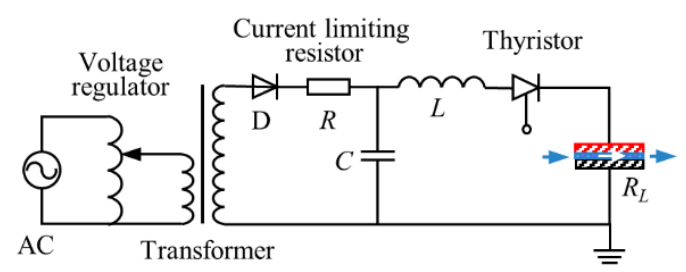

Fig. 2. Diagram of the circuit of pulsed power generator.

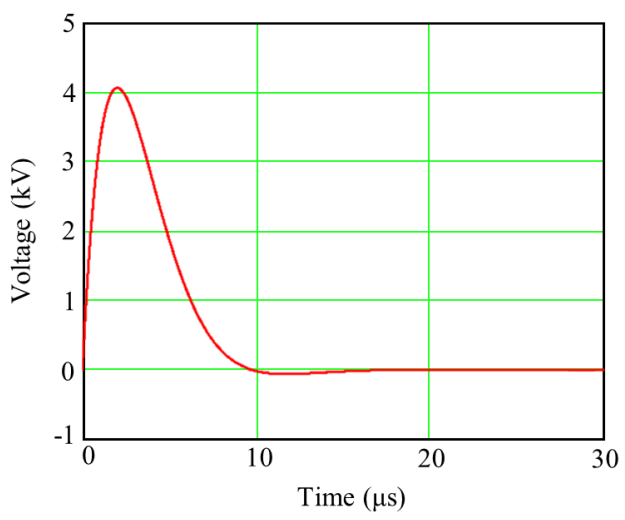

Fig. 3. Waveform of the pulse voltage applied to the PEF treatment chamber.

Another major component of the PEF system is the treatment chamber used to transfer voltage pulses to test object, shown as the load $R_{L}$ in Fig. 2 and therefore its equivalent electrical resistance was carefully considered before designing a matched chamber. As (3) shows, for a given conductivity $\sigma$ of the liquid flowing, the treatment chamber ohmical resistance $R_{S}$ is related to the distance between electrodes and the area of liquid contacting with electrodes. The resultant $R_{S}$ must be therefore as close as possible to $R_{L}$. To make it more suitable for the treatment. When the chamber was designed, there were other constraints considered such as the shape of electrodes, their configuration and the insulating materials.

$$
R_{S}=\frac{d}{\sigma A}
$$

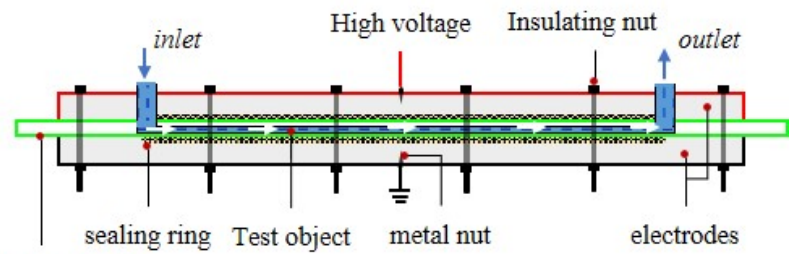

acrylic spacer

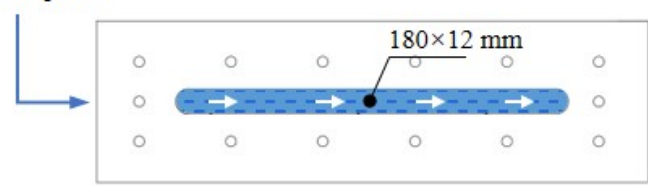

Fig. 4. Details of the PEF treatment chamber. 
Fig. 4 shows the cross-section of the PEF treatment chamber consisting of two parallel mounted stainless steel electrodes placed a distance $d=2.7 \mathrm{~mm}$ apart using an acrylic spacer, with the electrodes and the spacer fixed using plastic screws and nuts. The electrodes are $240 \mathrm{~mm}$ long and $49 \mathrm{~mm}$ wide. Nutrient solution flows through the channel (blue area in Fig. 4) in the acrylic spacer with a volume of $5.52 \mathrm{~mL}$.

\section{Energy consumption}

The energy consumption of a PEF treatment is one of the most important parameters which depends on the electric field strength and the length of the pulse, as well as the number of pulses ${ }^{[17]}$.

The energy delivered in each pulse is given by:

$$
W_{p}=\int_{0}^{t_{p}} u(t)^{2} / R_{L} d t
$$

where $u(t)$ is the time-varying voltage applied to the electrodes, $R_{L}$ is the resistance of the test object and $t_{p}$ is the pulse duration. The total applied energy is simply obtained by multiplying $W_{p}$ with the number of the pulses.

\section{Measurement of surviving spores and cells}

The number of surviving Fusarium oxysporum spores was evaluated by colony counting method and measured by plating $0.1 \mathrm{~mL}$ of each sample and serially diluted sample with a sterilized $0.9 \mathrm{wt} \% \mathrm{NaCl}$ aqueous solution onto PDA. This procedure was performed on a clean bench to prevent unwanted bacteria pollution. The plates were incubated at $28{ }^{\circ} \mathrm{C}$ for $36 \mathrm{~h}$. After the incubation, the plates with $30-300$ spores or CFU were chosen to count the colonies ${ }^{[18]}$. The detection limit was 1 spore $/ \mathrm{mL}$ or CFU/mL. The disinfection efficiency $\delta(\%)$ was calculated by $(5)$.

$$
\delta(\%)=\frac{N_{0}-N}{N_{0}} \cdot 100 \%
$$

where $N_{0}$ and $N$ are the colony numbers of Fusarium oxysporum before and after the PEF treatment, respectively.

\section{E. Measurement of inorganic compositions in the hydroponic nutrient solutions}

The same hydroponic nutrient solution as described above was used. The PEF treatment was performed applying a peak electric field strength $14 \mathrm{kV} / \mathrm{cm}$ with a flow rate of $0.3,1$ and $2 \mathrm{~L} / \mathrm{min}$, respectively, these being the most severe parameters of all the conditions tested. The concentrations of nitratenitrogen and available potash in the hydroponic nutrient solutions before and after PEF treatment were analyzed by a spectrophotometer (UV-3150) and an atomic absorption spectrophotometer (AA-7000), respectively.

All experiments were performed in triplicate.

\section{RESULTS AND DISCUSSION}

\section{A. Influence factors of PEF on the sterilization effect of Fusarium oxysporum in nutrient solution}

Many factors are affecting the efficiency of PEF treatment on microorganisms, with the most important being the peak electric field strength and the treatment time. In this study, the influence of these two factors on the sterilization efficiency is analysed in detail. The processing time varies with the flow rate and the effect of the flow rate on the disinfection efficiency is presented in Fig.5.

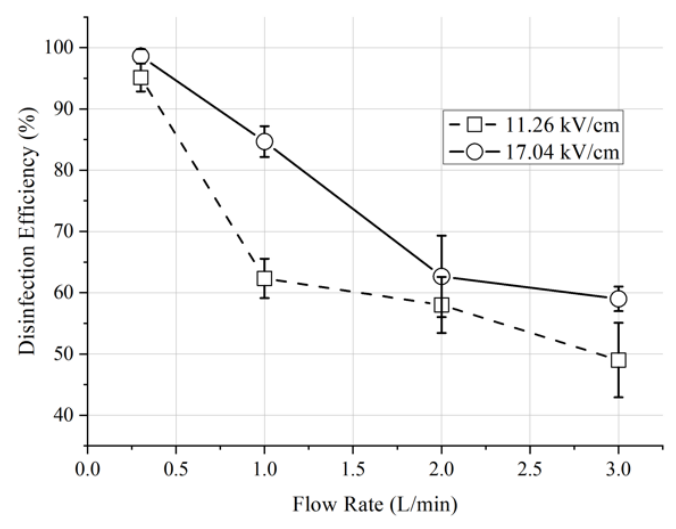

Fig. 5. Disinfection efficiency versus flow rate.

As Fig. 5 shows, the disinfection efficiency decreases with an increase of the flow rate. This is because the increase of flow rate decreases the number of pulses applied to the nutrient solution per unit time. When the peak electric field strength was $17.04 \mathrm{kV} / \mathrm{cm}$ and the flow rate was only $0.3 \mathrm{~L} / \mathrm{min}$, the disinfection efficiency of Fusarium oxysporum reached $99 \%$.

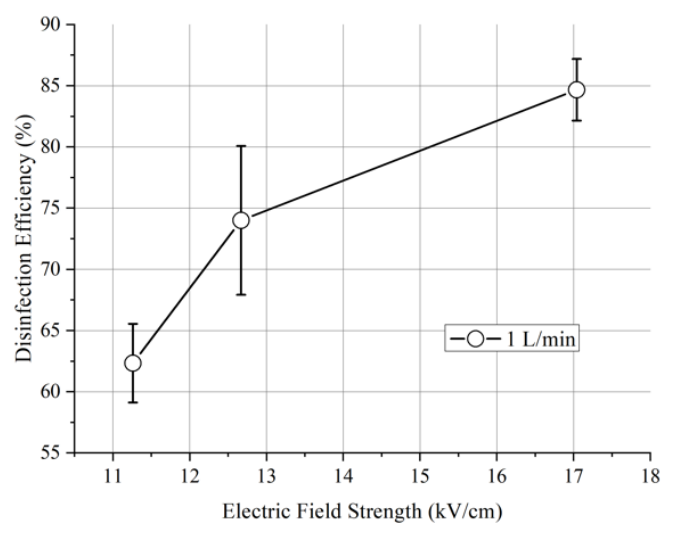

Fig. 6. Disinfection efficiency versus electric field strength.

As presented in Fig. 6, while maintaining the same liquid flow rate of $1 \mathrm{~L} / \mathrm{min}$, the disinfection efficiency of Fusarium oxysporum increases with the applied peak electric field strength. The above results are consistent with results from previous studies of application of PEF disinfection. Since the frequency of pulses in this work was only $50 \mathrm{~Hz}$, higher frequency and more powerful electric field strength are required to obtain a larger treatment capacity per unit time.

\section{B. Effect of PEF treatment on inorganic compositions in nutrient solution}

Fig. 7 shows the test results of the inorganic composition in nutrient solution after PEF treatment under different flow rates at a peak electric field strength of $14 \mathrm{kV} / \mathrm{cm}$. The results indicate the compositions of nitrate-nitrogen and available potash in the nutrient solution have a negligible change. This clearly demonstrates the advantage of the PEF treatment technique that can efficiently implement disinfection without obviously changing the nutrient solution, which is friendly to all practical soilless culture planting. 


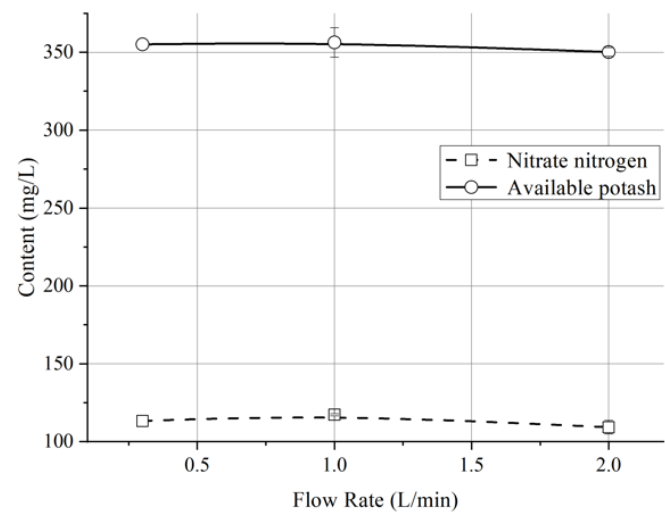

Fig. 7. The changes of compositions in nutrient solution after PEF treatment with a peak electric field strength of $14 \mathrm{kV} / \mathrm{cm}$.

\section{Analysis of energy consumption}

As can be seen from Fig. 8, the energy efficiency varied inversely to the strength of the peak electric field strength and positively proportional to the flow rate. For an applied peak electric field strength of $11.26 \mathrm{kV} / \mathrm{cm}$ and with the nutrient solution flow rate $0.3 \mathrm{~L} / \mathrm{min}$, the sterilization rate was $95 \%$ with the corresponding energy consumption being between $159.59 \mathrm{~L} / \mathrm{kWh}$ and $173.26 \mathrm{~L} / \mathrm{kWh}$.

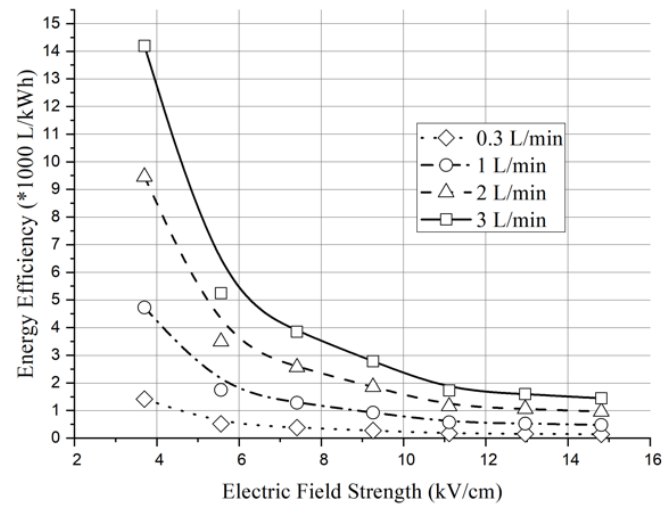

Fig. 8. Energy efficiency under different field strength and flow rate.

\section{CONCLUSION}

In this work, a prototype of a PEF disinfection was developed, which allows a continuous treatment of nutrient solution. Based on a highly efficient HV thyristor closing switch, the pulsed power generator was capable of stable operation at a pulsed repletion frequency of $50 \mathrm{~Hz}$. Significant disinfection efficiency of Fusarium oxysporum in the nutrient solution was observed during experiments. By applying peak electric field strength as high as $17.04 \mathrm{kV} / \mathrm{cm}$ while maintaining a flow rate of $0.3 \mathrm{~L} / \mathrm{min}$, the disinfection rate approached $99 \%$. Within the scope of this experiment, the inactivation effect of PEF on Fusarium oxysporum increased with the rise of the peak electric field strength and the decrease of flow rate. The results obtained are consistent with the studies of other scholars. Very importantly, after the PEF treatment there were no obvious changes in the composition of nitrate-nitrogen and available potassium in the nutrient solution. When the disinfection rate was $95 \%$, the energy efficiency was between $159.59 \mathrm{~L} / \mathrm{kWh}$ and $173.26 \mathrm{~L} / \mathrm{kWh}$. This work clearly demonstrates the feasibility and potentiality of continuous PEF disinfection equipment utilized for various future real soilless culture system applications.

\section{REFERENCES}

[1] S. Hosseinzadeh, Y. Verheust, G. Bonarrigo, and S. Van Hulle, "Closed hydroponic systems: operational parameters, root exudates occurrence and related water treatment," Reviews in Environmental Science and Bio/Technology, vol. 16, pp. 59-79, 2017.

[2] S. Yasui, S. Seki, R. Yoshida, K. Shoji, and H. Terazoe, "Sterilization of Fusarium oxysporum by treatment of non-thermalequilibrium plasma in nutrient solution," JAPANESE JOURNAL OF APPLIED PHYSICS, vol. 55, 2016.

[3] J. Mori and R. Smith, "Transmission of waterborne fish and plant pathogens in aquaponics and their control with physical disinfection and filtration: A systematized review," Aquaculture, vol. 504, pp. 380$395,2019$.

[4] A. Tsunedomi, K. Miyawaki, A. Masamura, M. Nakahashi, K. Mawatari, T. Shimohata, T. Uebanso, Y. Kinouchi, M. Akutagawa, T. Emoto, and A. Takahashi, "UVA-LED device to disinfect hydroponic nutrient solution," J Med Invest, vol. 65, pp. 171-176, 2018-01-20 2018.

[5] M. Tamaki, F. Kobayashi, H. Ikeura, and M. Sato, "Disinfection by Ozone Microbubbles Can Cause Morphological Change of Fusarium oxysporum f. sp. melonis Spores," Plant Pathol J, vol. 34, pp. 335-340, 2018-08-01 2018.

[6] W. Raza, N. Ling, R. Zhang, Q. Huang, Y. Xu, and Q. Shen, "Success evaluation of the biological control of Fusarium wilts of cucumber, banana, and tomato since 2000 and future research strategies," Critical Reviews in Biotechnology, vol. 37, pp. 202-212, 2017-01-01 2017.

[7] W. Nosir, "New technique for rose production in soilless culture system and disease reduction," Journal of Plant Nutrition, vol. 39, pp. 181-188, 2016-01-01 2016.

[8] D. Dannehl, I. Schuch, Y. Gao, S. Cordiner, and U. Schmidt, "Effects of hypochlorite as a disinfectant for hydroponic systems on accumulations of chlorate and phytochemical compounds in tomatoes," European Food Research and Technology, vol. 242, pp. 345-353, 2016.

[9] N. Igura, M. Fujii, M. Shimoda, and I. Hayakawa, "Inactivation efficiency of ozonated water for Fusarium oxysporum conidia under hydroponic greenhouse conditions.," Ozone: Science and Engineering, vol. Vol.26, pp. 517-521, 2004.

[10] W. Yiasoumi, L. Evans, and L. Rogers, Farm Water Quality and Treatment. NSW Department of Primary Industries, Orange, NSW, Australia, 2014

[11] K. SAITO, Y. NOZAWA, Y. MINAMITANI, and H. FUKATA, "Development of Nonthermal Sterilization Treatment System without Impacting to Enzyme in Vegetable Drink by Pulsed Electric Field," Electronics and Communications in Japan, vol. 101, pp. 85-92, 201801-01 2018.

[12] Tsong, "Electroporation of Cell Membranes," Biophysical Journal, vol. 60 (2), pp. 297 306, 1991

[13] U. Zimmermann, "Electrical breakdown, electropermeabilization and electrofusion," Reviews of physiology, biochemistry and pharmacology, vol. 105, p. 176, 1986-01-01 1986.

[14] Q. Wang, Y. Li, D. Sun, and Z. Zhu, "Enhancing Food Processing by Pulsed and High Voltage Electric Fields: Principles and Applications," Critical Reviews in Food Science and Nutrition, vol. 58, pp. 22852298, 2018-01-01 2018.

[15] C. Zhong, X. Guan, Z. Fan, W. Song, R. Chen, Y. Wang, X. Sun, and S. He, "Pulsed electric field disinfection treatment of Fusarium oxysporum in nutrient solution," Water Supply, vol. 19, pp. 2116-2122, 2019-11-01 2019.

[16] X. Huang, M. Sun, X. Lu, and S. Li, "Serial passage through resistant and susceptible cucumber cultivars affects the virulence of Fusarium oxysporum f. sp.cucumerinum," MicrobiologyOpen, vol. 8, p. e00641, 2018.

[17] F. De Vito, "Application of Pulsed Electric Field (PEF) Techniques in Food Processing,". vol. Ph.D. Course in Chemical Engineering, 2006.

[18] T. Sato, Y. Murakami and Y. Muramoto, "Estimation of liquid sterilization using critical voltage under high electric field pulses," IEEJ Transactions on Electrical and Electronic Engineering, vol. 14, pp. 1002-1007, 2019. 